\title{
Assignment of Dynamic Transmission Range Based on Estimation of Vehicle Density
}

\author{
Maen M. Artimy William Robertson William J. Phillips \\ Engineering Mathematics and Internetworking, Dalhousie University \\ Halifax, Nova Scotia, Canada, B3J 2X4 \\ artimym@dal.ca \\ bill.robertson@dal.ca \\ william.phillips@dal.ca
}

\begin{abstract}
Vehicular Ad Hoc Networks (VANET) have several characteristics that distinguish them from other ad hoc networks. Among those is the rapid change in topology due to traffic jams, which also disturbs the homogenous distribution of vehicles on the road. For this reason, a dynamic transmission range is more effective in maintaining connectivity while minimizing the adverse effects of a high transmission power.

We provide a relationship that allows vehicles to estimate the local density and distinguish between two phases of traffic, freeflow and congested traffic. The density estimate is used to develop an algorithm that sets a vehicle transmission range dynamically according to local traffic conditions.

Simulations of various road configurations show that the algorithm is successful in maintaining connectivity in highly dynamic networks.
\end{abstract}

\section{Categories and Subject Descriptors}

C.2.1 [Network Architecture and Design]: Wireless communication;

\section{General Terms}

Design, Performance.

\section{Keywords}

Ad Hoc Networks, Connectivity, Density estimation, Inter-vehicle communications, Transmission range, VANET.

\section{INTRODUCTION}

Vehicular Ad Hoc Networks (VANET) have several characteristics that distinguish them from other mobile ad hoc networks. Common mobility models of mobile ad hoc networks (MANET) allow node mobility (determined by speed or pause time) to be considered independently of their density in protocols development and performance evaluation. In contrast, car-

Permission to make digital or hard copies of all or part of this work for personal or classroom use is granted without fee provided that copies are not made or distributed for profit or commercial advantage and that copies bear this notice and the full citation on the first page. To copy otherwise, or republish, to post on servers or to redistribute to lists, requires prior specific permission and/or a fee.

VANET'05, September 2, 2005, Cologne, Germany.

Copyright 2005 ACM 1-59593-141-4/05/0009...\$5.00. following models $[18,24]$ show that the average speed of vehicles is a function of vehicle density. This provides the motive to use density as a single metric describing the nodes' mobility in VANETs.

Node density has a great impact on the performance of ad hoc networks by influencing factors such as capacity, routing efficiency, delay, and robustness. Waves of traffic jams, whether caused by constraints in the transportations network, traffic controls, or driving fluctuations, cause the network's density to vary from one location to another; thus disturbing the homogenous distribution of nodes. Moreover, the abrupt and frequent change in density creates a highly dynamic topology. This topology change would cause severe degradation to the network's performance (increased collisions and interference, excessive broadcasts, too many routing paths, etc.) if protocols in VANETs were not designed to handle such conditions.

Controlling the communication range by adjusting the transmission power can be used to mitigate the adverse effects of high density condition. The choice of the communication range has a direct impact on a fundamental property of an ad hoc network, the connectivity. In a VANET, a static transmission range cannot maintain the network's connectivity due to the nonhomogenous distribution of vehicles and rapid change of traffic conditions. It is shown in $[10,23]$ that a dynamic transmission range is needed to maintain connectivity in non-homogeneous networks to take advantage of power saving and increased capacity.

In this paper we derive a relationship that allows a vehicle to estimate the local density. This relationship is used to set the vehicle's transmission range dynamically. The result is a highly dynamic transmission range algorithm that requires no external information (such as vehicle position). Moreover, there is no communication overhead involved since the algorithm uses only the vehicle's internal state to determine the transmission range. The algorithm is transparent to the data communications protocols. Therefore, it can be integrated with existing systems with little or no change to the latter. The algorithm is highly adaptable to traffic conditions (density and speed) that may change in a very short time due to traffic jams.

The paper is organized into nine sections as follows: The related work is summarized in Section 2. In Section 3, a brief introduction to traffic theory is provided. Section 4 describes the road configurations used in the simulations and the communications model. In section 5, we show the effect of density on the minimum transmission range. The density 
estimation scheme is provided in Section 6. Section 7 describes the dynamic range algorithm followed by its performance evaluation in Section 8. The summary and conclusions are provided in Section 9.

\section{RELATED WORK}

Vehicular ad hoc networks can be considered examples of 1dimensional networks, where nodes (vehicles) are placed along a line. Connectivity in these networks is studied extensively in the literature. A 1-D network is considered connected if there is no gap wider than the transmission range between any pair of successive nodes. Santi and Blough estimate the lower and upper bounds for the transmission range in [27] and Desai and Manjunath [7] provide a probability for gap existence among nodes.

In networks of infinite size, the transmission range is related to node density rather than the line's length [6]. Connectivity in infinite networks is limited to short range communications and a large-scale ad hoc network is not feasible because it is almost surely divided into an infinite number of partitions. The same conclusion applies also to strip networks (networks of infinite length in one dimension and a finite length in the other) [8].

The work presented in [6-8,27] assumes a homogenous distribution of nodes. It will be shown in Sections 3 and 5 that this assumption is not always valid in VANETs due to traffic jams and bottlenecks.

Our approach to dynamic range assignment in Section 7 is closely related to a class of topology control algorithms that control a node's degree (number of neighbours) by adjusting the transmission power [15]. These protocols depend on an exchange of information such as in [17] or on some protocol feature as in [3]. Our algorithm, on the other hand, depends only on a vehicle's mobility and does not require the exchange of messages.

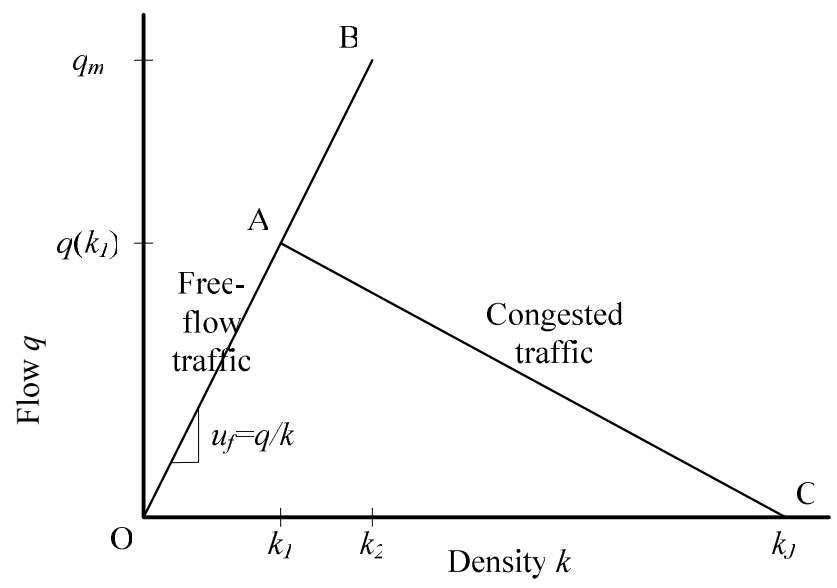

Figure 1 Flow-density relationship.

Many studies in VANETs focus on free-flow traffic in their design and analysis of new protocols (e.g. $[5,14,26])$. The studies that investigate connectivity either analytically or using simulations also set the traffic conditions to free flow $[9,25]$. This choice allows for the greatest flexibility in controlling each of the vehicle traffic parameters (speed, flow, and density) independently. We deal with the entire density range and show that mobility and speed cannot be always distinguished from density.

\section{INTRODUCTION TO TRAFFIC THEORY}

Traffic flow theories explore relationships among three main quantities; vehicle density, flow, and speed. The flow $q$, measures the number of vehicles that pass an observer per unit time. The density $k$, represents the number of vehicles per unit distance. The speed $u$, is the distance a vehicle travels per unit time. The units of these quantities are usually expressed in (veh/hr/lane), (veh/km/lane), and $(\mathrm{km} / \mathrm{hr})$, respectively.

In general, traffic streams are not uniform, but vary over both space and time. Therefore, the quantities $q, k$, and $u$ are meaningful only as averages or as samples of random variables [12]. The three quantities are related by the so-called Fundamental Traffic Flow relationship [11],

$q=u \cdot k$

Several theories attempt to define the relationships among each pair of variables in (1), but no single theory provides the complete picture. The following subsections provide a brief introduction to principles most relevant to the scope of this paper.

\subsection{Speed-Density Relationship}

Car-following models [24] provided an early means to describe the speed-density relationship. These models apply to single-lane, dense traffic with no overtaking allowed. They also assume that each driver reacts in some specific fashion to a stimulus from the vehicle(s) ahead or behind. The models do not apply in low densities where interactions between vehicles disappear. The basic equation describing a car-following model is [4]:

Response $=$ Sensitivity $\times$ Stimulus

The response may be interpreted as the acceleration of the following vehicle and the stimulus is the relative speed or distance between the following vehicle and the leading vehicle.

A class of car-following models assumes that drivers maintain constant time headway between vehicles. The model proposed by Pipes [21] results in the following speed-density relation [11,21]:

$u=\lambda\left(\frac{1}{k}-\frac{1}{k_{J}}\right)$

where $\lambda$ measures the sensitivity of the vehicle interaction and $k_{J}$ is the maximum vehicle density at traffic jam.

\subsection{The Fundamental Diagram of Road Traffic}

The Fundamental Diagram of Road Traffic describes the flowdensity relationship. A typical $q-k$ relationship follows the general shape of Figure 1. The figure shows that the flow is zero when there are no cars on the road, and also when there is a complete traffic jam at maximum density, $k_{J}$. The shape of Figure 1 suggests that there are two $q-k$ regimes. The left branch (OA) of the relationship represents free-flow traffic at densities below $k_{1}$. In the free-flow phase, interactions between vehicles are rare because 
of the low density. As a result, vehicles can travel at free-flow speed $u_{f}$, determined by the slope of the left branch, $u_{f}=q / k$. At densities above $k_{2}$, traffic become congested and hindered by traffic jams. The $q-k$ relationship of the congested traffic is represented by the right branch (AC). Empirical evidence and traffic simulations suggest that traffic may also be in a coexistence phase. In densities between $k_{1}$ and $k_{2}$, drivers may accept shorter headway (time gap) between vehicles; thus, achieving the maximum flow $q_{m}$, at the critical density, $k_{2}$. High fluctuations of speed in this traffic phase may cause a break down in the flow and derive the traffic into the congested phase.

At densities higher than $k_{1}$, speed can be expressed as a function of density as in (3). By substituting (3) in (1) and accounting for low-density traffic, a piecewise linear $q-k$ relationship can represent the OAC triangle of Figure 1 [11],

$$
q=\min \left(u_{f} k, q_{1}\left(1-\frac{k}{k_{J}}\right)\right)
$$

where $q_{1}=q\left(k_{1}\right) \approx \lambda$.

\subsection{Traffic Jams and Phase Transition}

Traffic jams are caused by geometric constraints, such as a red light, an accident, or an access ramp. These scenarios can be described by a standard queuing system where the jam at the bottleneck will grow spatially backward if the arrival rate (inflow) is greater than the service rate (outflow) of the bottleneck. The spatial growth can be described also by the theory of kinematic waves [16].

It is also suggested that waves of traffic jams may occur without the presence of obvious constraints but mainly due to fluctuation (noise) in speed. These fluctuations may be caused by bumps, curves, lapses of attention, and different engine capabilities. In moderate traffic flow, a noise of high amplitude may cause the traffic to become unstable and traffic jams start to appear [18]. This type of traffic jams can be described by the theory of kinematic waves, and has been studied extensively using Cellular Automata (CA) models [13,19].

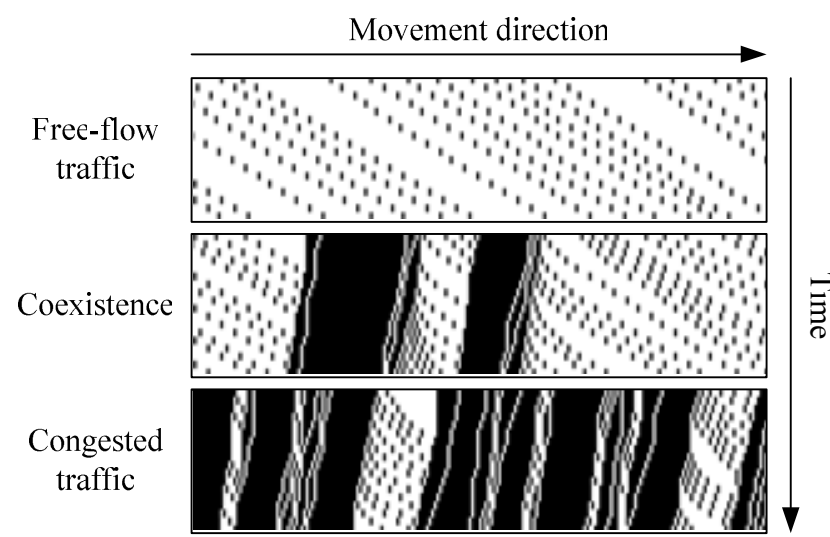

Figure 2 Space-time diagram illustrating phase-transition from free-flow to congested traffic.

The transition from free-flow traffic to congested traffic can be described with the help of the space-time diagram of Figure 2. A space-time diagram shows vehicle positions as they would appear in a series of aerial photographs taken at fixed time intervals of a road section and lined-up vertically according to their time index. A vehicle moving at constant speed will appear as a series of dots arranged diagonally in the space time diagram, while a stationary vehicle will appear as a column of dots.

The top section of Figure 2 shows the traffic flow at density slightly lower than $k_{1}$ (of Figure 1) where a vehicle can travel freely without influence from other vehicles. The middle section shows traffic whose density is in the range $\left[k_{1}, k_{2}\right]$. At this density, drivers may adapt to the dense traffic by slowing down and maintaining a minimum safety distance. However, high fluctuations in speed by a lead vehicle may break the flow and create traffic jams. A traffic jam appears in the middle section of Figure 2 as dark clusters of dots moving backward against the traffic direction. Vehicle that are not caught in jams are traveling in free-flow traffic. Traffic jams grow as density increases and merge with other traffic jams, as shown in the bottom section of Figure 2. Ultimately, the entire road section is occupied with one wide traffic jam.

\section{VEHICLE NETWORK SIMULATION}

The following two subsections describe the simulation setup used for this work.

\subsection{Vehicle Mobility}

Vehicle movement traces are generated using a traffic microsimulator based on a CA model [2]. Three road configurations are used for the experiments described later to represent distinct traffic situations. Traffic density is increased gradually in two configurations so that traffic conditions can change gradually from free-flow to congested traffic. In the third configuration, an intersection controlled by traffic lights is used to cause a rapid change in vehicle density across the intersection.

In single-lane roads with no overtaking, vehicles will travel at the speed of the slowest vehicle regardless of their maximum speed capabilities. Therefore, all vehicles have the same maximum speed of $135 \mathrm{~km} / \mathrm{hr}$ in simulations of single-lane roads while the three-lane road configuration includes vehicles of various maximum speeds. Note that the maximum speeds are meaningful only as free-flow speeds. In higher densities, vehicles travel at lower speed, which is unrelated to their maximum speed.

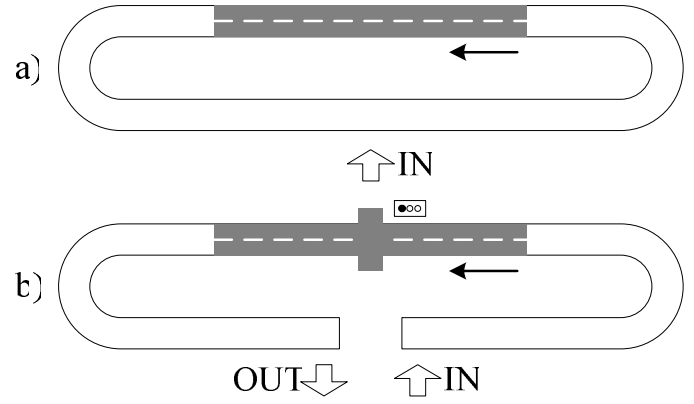

Figure 3 Road configurations: a) Racetrack; b) Intersection. The data collection is limited to the shaded area.

In the first configuration, vehicles travel in a single-lane road where overtaking is not allowed. The road is in the form of a racetrack of $7.5 \mathrm{~km}$ length (Figure $3 \mathrm{a}$ ). Vehicles enter from a 
parking facility at a rate (flow) of $60 \mathrm{veh} / \mathrm{hr}$ and continue to travel around the track indefinitely, which causes vehicle density to increase until the jam density is reached and no more vehicles can enter.

The racetrack road in the second configuration has three lanes, which allows the vehicles to change lanes in order to pass each other. Vehicles in this configuration are divided into three classes. Slow vehicles compose $15 \%$ of all vehicles and their maximum speed is set to $81 \mathrm{~km} / \mathrm{hr}$. Another $15 \%$ of vehicles can travel at maximum speed of $135 \mathrm{~km} / \mathrm{hr}$. The remaining vehicles have a maximum speed of $108 \mathrm{~km} / \mathrm{hr}$. This distribution reflects the $85^{\text {th }}$ percentile rule that is used as a guide to set the speed limit on highways so that $85 \%$ of vehicle will travel below the limit. The flow rate of vehicles is set to $300 \mathrm{veh} / \mathrm{hr} / \mathrm{lane}$.

The third configuration (Figure $3 b$ ) consists of a single-lane road with a traffic signal controlled intersection in the middle. Vehicles enter from a parking facility at one end of the road, at rate of $1800 \mathrm{veh} / \mathrm{hr} / \mathrm{lane}$, and exit from the other end. The traffic signal has a cycle of one minute divided equally between red and green. In this open-loop road, a traffic jam occurs behind the red light. The combination of traffic inflow and the red light duration ensures that the traffic jam is created periodically throughout the simulation.

In racetrack configurations, data collection is restricted to a segment of length $L \approx 1 \mathrm{~km}$ located at the furthest distance from of the entry point so that measurements are not effected by vehicle interactions at that point. In the intersection configuration, data is collected in two $1 \mathrm{~km}$ segments immediately before and after the intersection. The segment is chosen to be of length comparable to the maximum transmission range of recent proposed standards such as Dedicated Short Range Communications (DSRC). The segment also represents a proper sized network for some applications, such as safety messaging.

In all experiments, data is collected from 20 simulation runs. Depending on the configuration, simulation time ranges form 25,000 to 60,000 seconds.

\subsection{Communications Model}

The VANET is represented as a graph $G(V, E)$, where a set of vertices $V$ represents vehicles, and a set of edges $E$ represents direct communication links. In the simple communication model that is used here, a communication link, $e_{i, j}=\left(v_{i}, v_{j}\right)$ exists if and only if the Euclidean distance between vehicles $v_{i}$ and $v_{j}$ is less than or equal to the shortest transmission range between them, i.e,

$$
E=\left\{\left(v_{i}, v_{j}\right) \in V^{2}\left|x_{i}-x_{j}\right| \leq \min \left(r_{i}, r_{j}\right)\right\}
$$

where $x_{i}, r_{i}$ are the position and transmission range of the node $v_{i}$, respectively. Equation (5) implies that the graph $G(V, E)$ is a undirected graph.

\section{MINIMUM TRANSMISSION RANGE AND CONNECTIVITY IN VEHICLE NETWORKS}

Considerable effort has been directed towards the study of connectivity in 1- and 2-dimensional ad hoc networks. A major factor of maintaining connectivity in these networks is the choice of the minimum (critical) transmission range (MTR) that guarantees that a network is connected (i.e. each node has a least one path to every other node).

In this section, we illustrate the effect of vehicle traffic characteristics on the MTR when density is changed from freeflow to a total traffic jam. We should emphasize that increasing the density not only increases the number of vehicles on the road but also creates traffic jams and lowers the average speed of vehicles when the density is higher than some critical density.
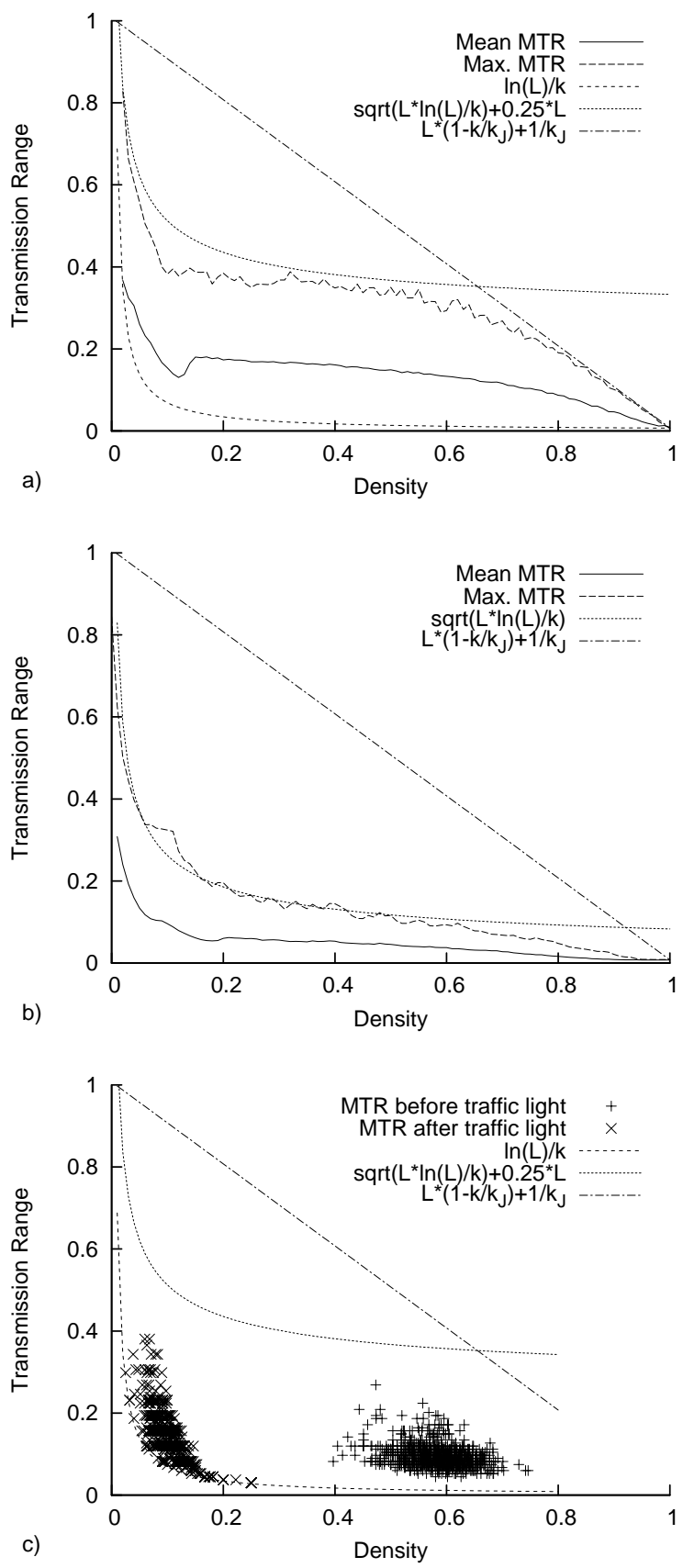

Figure 4. MTR statistics in a) 1-lane road, and b) 3-lane road. c) MTR values near a traffic light $(\mathbf{9 9 \%}$ of points are removed to reduce the clutter) 
In general, the MTR is determined by constructing a minimum spanning tree (MST) among all vehicles in the network. The MTR is the longest edge in the MST [20]. In a single-lane VANET, the MTR is the widest gap between any two consecutive vehicles.

Simulations are used to determine the MTR needed to connect the wireless network among vehicles in the road configurations of Section 4.1. The MTR is determined in every simulation time-step and stored along with the vehicle density. The data collected from the simulations is classified into 100 density intervals that cover the entire density range. The basic statistics of the MTR are calculated within each energy interval to plot MTR vs. density values for each scenario.

The solid line in Figure 4a shows the mean value of MTR vs. density in the single-lane road configuration. At low densities, the MTR decreases as density increases. At the transition point between free-flow and dense traffic, the MTR increases again then resumes its decline until it reaches its minimum value at total traffic jam. Figure 4a indicates that, despite the higher density, a longer MTR is needed to connect the vehicles near the phase transition point of $k \sim 1 / 6$.

The sudden increase in MTR near the point of phase transition is caused by the creation of traffic jam waves that result in some vehicles being clustered in small areas while other are spread in less dense traffic, which has the effect of disturbing the homogenous distribution of vehicles (as shown in the middle section of Figure 2). As density increases, traffic jams increase in size and merge with others until the entire road is occupied by one large traffic jam. Consequently, the MTR decreases until it reaches its minimum value of $1 / k_{J}$ (the distance from front-bumper to front-bumper between two vehicles).

The plot of average MTR and the average maximum MTR are compared to three other relations: The curve $\ln (L) / k$ [5] provides the theoretical lower bound of MTR. The absolute maximum MTR, $r_{\max }$, for a network of finite length, $L$, is needed if all but one vehicle are packed at distance of $1 / k_{J}$ from each other in one side in the network while the remaining vehicle is located at the opposite side; therefore,

$r_{\max }=L\left(1-\frac{k}{k_{J}}\right)+\frac{1}{k_{J}}$

The graph in Figure 4a shows that the average maximum MTR can be approximated by shifting up the upper bound in [27] by some factor, $a L$,

$r \leq \sqrt{L \ln (L) / k}+a L$

where $a=0.25$.

In the multi-lane configuration, vehicle locations are more homogenously distributed along the road's length as the number of lanes increases. As a result, Figure $4 \mathrm{~b}$ shows a slight increase in the mean MTR value near the critical density (which is now moved to $k \sim 1 / 4$ due to the lower average free-flow speed). The MTR is lower than the single-lane case by a factor of 3 due to the increase in the number of vehicles by the same factor to achieve the same density (per lane). The average maximum MTR can be approximated by (7) with $a=0$.
Instead of the MTR statistics, Figure 4c shows the actual MTR values to emphasize the effect of the abrupt change in traffic conditions from dense traffic behind a red light to free-flow a few seconds after the light turns green. This situation is illustrated in Figure $4 \mathrm{c}$ by the presence of two clusters of MTR values. The clusters at high and low densities correspond to vehicles behind and after the intersection, respectively. The gap between the clusters indicates that the transition between the two densities is abrupt and takes the form of a shock wave [16].

\section{DENSITY ESTIMATION}

In this section, it will be shown that a vehicle can estimate the local vehicle density using its own movement pattern. This estimate is based on traffic theory and the phase transition analogy described in Section 3.

\subsection{Density Estimate}

Traffic Flow theories suggest that the average vehicle speed can be expressed as a function of density,

$u=f(k)$

The fraction of vehicles stopped in traffic $f_{s}$, is related to the average speed of vehicles (including the stopped vehicles) [1],

$u=u_{f}\left(1-f_{s}\right)^{n+1}$

where $n$ is a parameter that indicates the quality of service in the transportation network.

The value of $f_{s}$ can be measured by an external observer counting the number of vehicles in the traffic. The two-fluid theory relates the time a test vehicle circulating in a network is stopped, $T_{s}$, to the average fraction of vehicles stopped, $f_{s}$ during the same period, $T[1]$,

$f_{s}=T_{s} / T$

Equation (10) represents an ergodic principle embedded in the model, i.e., the network conditions can be represented by a single vehicle appropriately sampling the network.

Given a $u-k$ relationship such as (3), a normalized density value $k^{\prime}$, is given by

$k^{\prime}=\left[u^{\prime} / \lambda^{\prime}+1\right]^{-1}$

where $k^{\prime}=k / k_{J}, u^{\prime}=u / u_{f}$ and $\lambda^{\prime}=\lambda /\left(u_{f} k_{J}\right)$.

From (9), and (10), the normalized average traffic speed is:

$u^{\prime}=\left(1-T_{s} / T\right)^{n+1}$

Equation (12) can be substituted in (11) to provide the means for a vehicle to estimate the density of the surrounding traffic by monitoring its stationary time. We denote the density estimate by $K$, therefore,

$K=\left[\left(1-T_{s} / T\right)^{n+1} / \lambda^{\prime}+1\right]^{-1}$ 
Equation (13) inherits the limitations of the car-following model, which means that it cannot provide an estimate of density in freeflow traffic where there are no interactions between vehicles.

Before estimating vehicle density using (13), the values of $n$ and $\lambda$, and $T$ must be determined. Both $n$ and $\lambda$ reflect the traffic service level of the road and can be determined statistically. Our simulations show that, in highway scenarios, $n \sim 0$ and $1 / \lambda \sim 2 s$, which is linked to the safety time headway between vehicles that results in a flow $\left(q\left(k_{1}\right)\right.$ in Figure 1) of just below 1800 (veh/hr/lane).

The choice of the window size, $T$, depends highly on the rate of change in traffic conditions; a small value of $T$ results in a noisy estimate, while a large $T$ increases the estimate lag beyond usefulness. The simulations show that a choice of $T=10 \mathrm{~s}$ results in a high correlation between the actual density and its estimate.

\subsection{Order Parameter}

In physics, phase transition is characterized by an order parameter. A substance in an ordered state has an order parameter of non-zero value. The order parameter takes the value of zero in the disordered state. A similar concept can be used to describe the state of vehicular traffic. Vehicles in a traffic jam can be compared to a liquid with an order parameter of a value equal to the fraction of stopped vehicles, $f_{s}$. Traffic enters a disordered state when more vehicles move freely, similar to gas molecules, and the order parameter approaches zero $[18,22]$.

In free-flow traffic, all vehicles are moving $\left(f_{s}=0\right)$. Once a traffic jam is created, vehicles stop when they join the traffic jam at its end while other vehicles at the front accelerate away from the traffic jam. From an observer's perspective, there is always a fraction of vehicles which are completely stopped $\left(f_{s}>0\right)$.

From a single vehicle perspective, only the presence of a local order (a local traffic jam) is detectable if $T_{s} / T$ is used. This provides useful information since a vehicle in a VANET is most affected by its local conditions (e.g. in medium contention). A vehicle may use the value of $T_{s} / T$ directly to detect whether it is traveling in free-flow or congested traffic.

\subsection{Evaluation}

Simulation of Section 4.1 road configurations are used to determine whether equations (10) and (13) can provide a reasonable indication of change in traffic conditions and estimate the density. Vehicles calculate the ratio $T_{s} / T$ and $K$ in every simulated time step. This information is later compared with the actual density from an observer's perspective. Since vehicle density changes over space and time, the measure of the density over the entire road segment does not reflect the local traffic conditions experienced by vehicles. Therefore, to measure local densities, the entire road segment of $1 \mathrm{~km}$ is divided into short sections of $150 \mathrm{~m}$ length each.

Figure 5 shows a relationship between vehicle density in each section and the average value of $T_{s} / T\left(=f_{s}\right)$ calculated by the vehicles that pass through that section. The plot corresponding to the 1-lane road shows a sudden increase in the value of $f_{s}$ at point near $k=1 / 6$ where the phase change occurs. Note that this is the point where the MTR increases in Figure 4a. Recall that in the 3lane configuration, simulation setup sets the maximum speed of most vehicles lower than that of the 1-lane configuration. As a result, the slope of the free-flow branch of the $q-k$ relation (Figure 1 ) is lower and the critical density occurs at higher density than that of the 1-lane configuration, $k=1 / 4$. These observations support the discussion of the previous section; the value of $f_{\mathrm{s}}=T_{s} / T$ can be used as an indicator of the existence of free-flow condition when $f_{s}=0$.

Figure 6 shows that the $K-k$ relation deviates from a straight line at densities within the free-flow range $(k<1 / 6$ for 1-lane configuration and $k<1 / 4$ for 3-lane configuration). Within this range, equation (13) has a constant value of $\sim 0.1$ when $T_{s}=0$ (the vehicle is in constant motion). At higher densities, the estimate $K$ approaches the $K=k$ line in both road configurations.

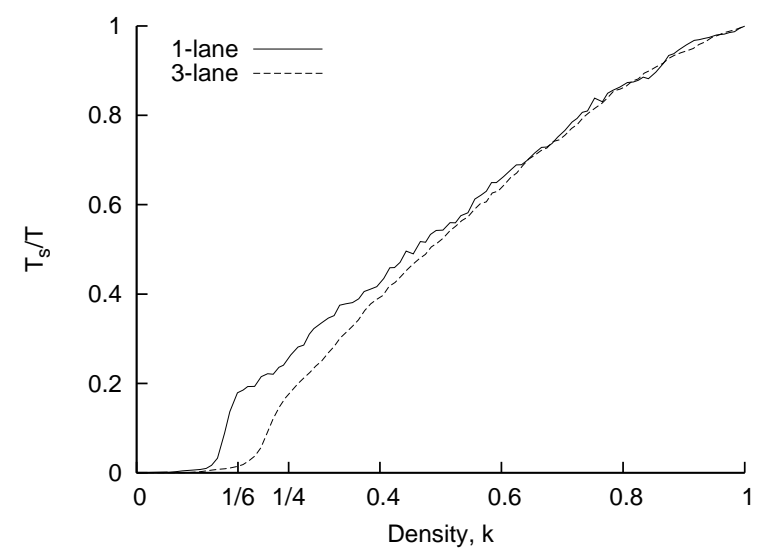

Figure 5. Order parameter vs. vehicle density in 1- and 3-lane roads.

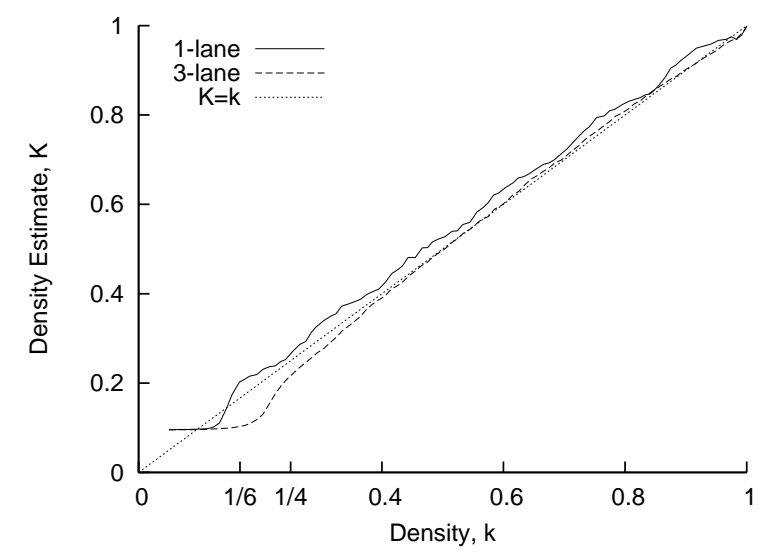

Figure 6. Density estimate, $K$, vs. actual density, $k$, in 1- and 3lane roads.

\section{DYNAMIC TRANSMISSION RANGE ALGORITHM}

It can be concluded from Figure 4 that if all vehicles had to use a common static transmission range for communication, the range should keep the vehicular network connected in all traffic conditions. This can be achieved only if the transmission range is high enough to accommodate conditions such as free-flow traffic and traffic across intersections. 
Alternatively, estimation of traffic density provides the necessary means to develop an open-loop power control algorithm to set a vehicle's transmission range dynamically as traffic conditions change. In its basic form, the algorithm maps the time-varying values of $T_{s} / T$ into a transmission range, $r$ at regular time intervals,

$$
r=g\left(T_{s} / T\right)
$$

The pseudo code shown in Figure 7 gives one implementation of the mapping function (14). The transmission range is set to its maximum level in free-flow traffic. In dense traffic, $r$ is determined by the minimum of equations (6) and (7). In general, $g($.$) may depend on other factors such as the desired level of path$ redundancy or the percentage of equipped vehicles. The choice of the maximum transmission range in free-flow is due to two reasons: 1) estimation of density within the free flow traffic range is difficult, but it is easy to detect the free-flow phase; 2) it is expected that distance between vehicles in free-flow is long; therefore a longer than optimal transmission range will not have the same adverse affects as in dense network and can help maintain the network stability.

Note that a practical algorithm would determine a power level instead of a transmission range. The power level may take any value in a set of possible power levels available to the wireless interface. We deal with the transmission range directly for an easy comparison with the network's length and the distance between vehicles.

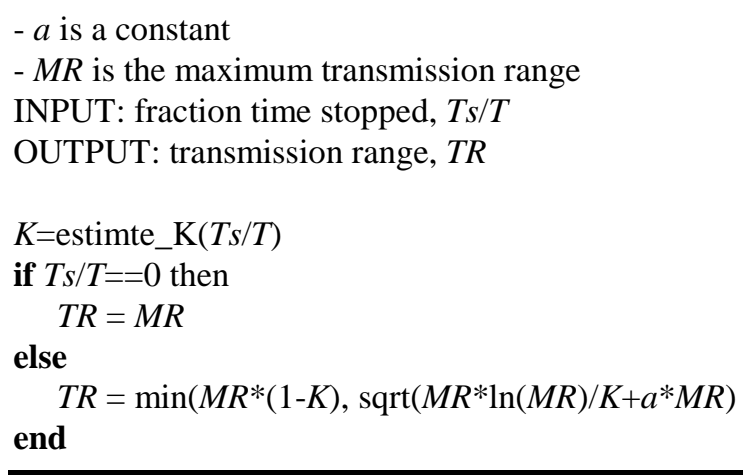

Figure 7. Dynamic transmission range algorithm.

\section{PERFORMANCE EVALUATION}

In this section we present several simulation results to demonstrate the effectiveness of the dynamic transmission range algorithm. We use the following metrics in the evaluation:

1. Number of network partitions: this is used to measure the network connectivity; a connected network consists of one partition.

2. Average transmission range: a smaller value of the transmission range implies less power level and better network spatial reuse.

The core algorithm described in Figure 7 is evaluated using simulations of the three road configurations described in Section 4.1. During the simulation, each vehicle estimates the local density and applies the algorithm to determine its own transmission range.
To count the number of partitions in the network: 1) A MST is constructed within the measurement section(s) of the road in the same way used to determine the MTR in Section 5. The edges of the MST represent the minimum distance between any two vehicles in the network. 2) Each edge in the MST is checked; if the edge does not satisfy equation (5), the network is partitioned at that point and the number of partitions is increased by one. Note that the procedure results in a network in which all communication links are bidirectional.
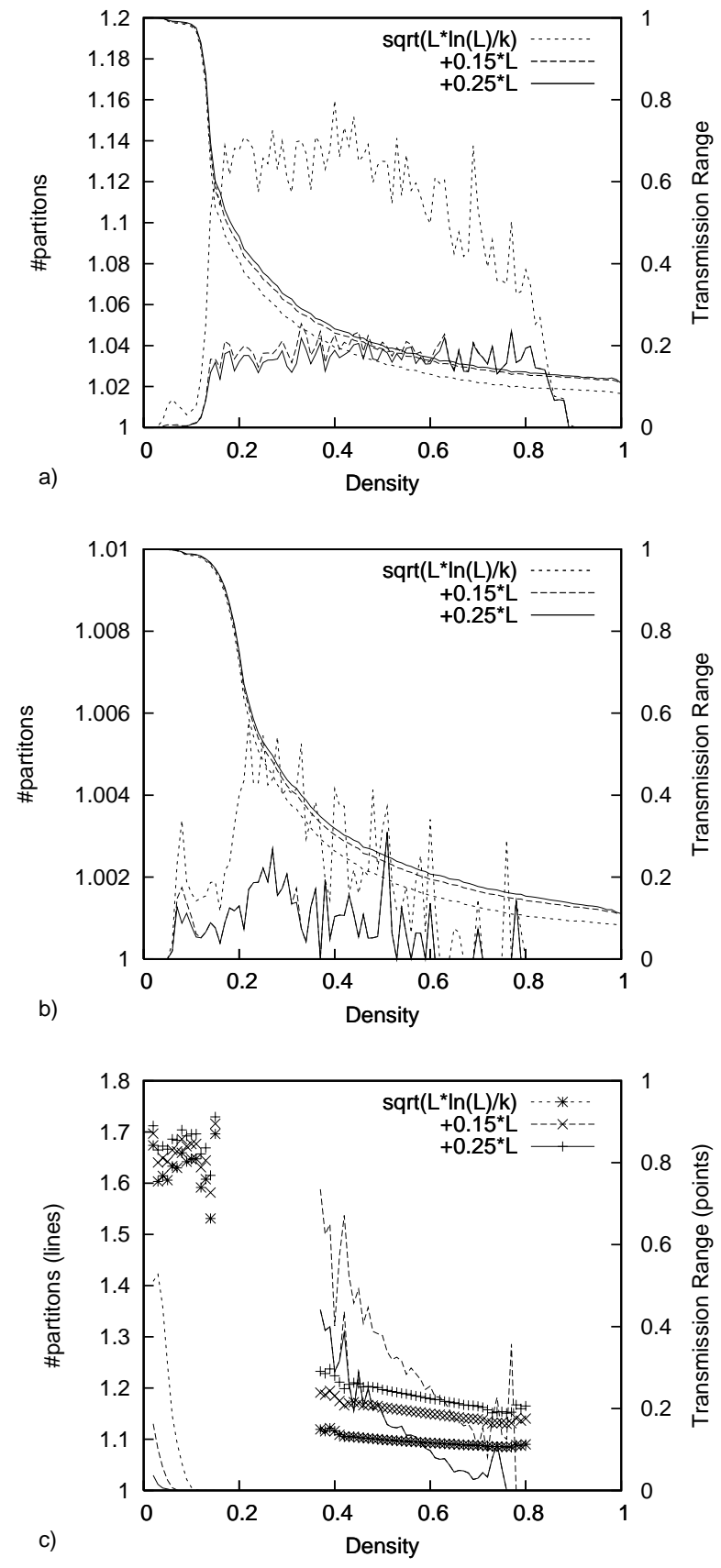

Figure 8. Vehicle density vs. number of partitions and average transmission range in a) 1-lane racetrack, b) 3-lane racetrack, and c) signalized intersection. 
Figure 8 shows the average number of partitions and the average transmission range along the density range in different road configurations. The different line types correspond to different choices for the parameter $a$ in equation (7), which sets the transmission range in the dense traffic. Figure $8 \mathrm{a}, \mathrm{b}$ show that the average number of partitions remains very close to one in racetrack scenarios. The transmission range drops quickly, near the critical density to approximately $10 \%$ of the maximum range while maintaining (or improving) the level of connectivity in racetrack configurations.

The figures also show the effect of changing the upper bound of the transmission range by shifting it up by a factor of $a L$. While there is little difference between the choice of $0.25 \mathrm{~L}$ and $0.15 \mathrm{~L}$, the number of partitions increases noticeably at $0 L$. The latter sets the upper bound below the mean MTR of the single-lane road and at roughly the mean MTR in the multi-lane road (Figure 4a,b). This indicates that the vehicles are able to set their transmission range very close to the optimal value in dense traffic.

The intersection scenario represents a challenging case for the dynamic transmission range algorithm since the network's condition changes rapidly across the traffic light, which requires fast switching of the transmission range from low (while vehicles are slowing or stopped) to high (while passing the intersection). The figure shows a higher number of partitions across the density range, which can be attributed to the slower reaction to the change in traffic condition. Nevertheless, Figure 8c shows that the average number of partitions remains low.

A closer look reveals that, when the lowest upper bound $(0 L)$ is used, the network behind the traffic light is connected $\sim 80 \%$ of the time, while the network ahead of the traffic light remains connected $\sim 96 \%$ of the time. The networks are partitioned into two partitions in $\sim 16 \%$ and $\sim 4 \%$ of the time, respectively.

\section{SUMMARY AND CONCLUSIONS}

A Vehicular Ad Hoc Network (VANET) is affected by the characteristics of traffic flow. Vehicle density influences the average speed of vehicles; thus, affecting the network's mobility. The density also affects the network topology by creating a nonhomogenous distribution of vehicles due to traffic jams. Traffic theories show that traffic jams are caused not only by constraints in the transportation network but also by fluctuations in driving in dense traffic. The effect of these traffic jams on VANET is an abrupt change in network topology.

In this paper, we propose a relationship to estimate the local density for each vehicle based on its movement pattern. This estimate can be used by many VANET protocols to set the parameters that depend on traffic conditions (e.g., Hello interval).

We used the density estimate to set the vehicle transmission range dynamically according to its local density. Simulations show that the dynamic transmission range algorithm is effective in maintaining a high degree of connectivity in road configurations where the network topology changes rapidly.

The proposed scheme has several advantages. The algorithm does not require any global information such as vehicle locations, nor does it require any exchange of information among vehicles. Moreover, the algorithm inherently adapts to vehicles mobility.
The algorithm is considered transparent to communications protocols, which allows its use in combination with other topology control protocols to achieve application-specific goals, or to provide an initial estimate of transmission range before further refinement.

A future study will involve a comparison of VANET performance using the dynamic range algorithm and other topology control algorithms that are based on message-exchange schemes.

\section{REFERENCES}

[1] Ardekani, S. and Herman, R. 1987. Urban network-wide traffic variables and their relations. Transportation Science, $21,1,1-16$.

[2] Artimy, M. M., Robertson, W. and Phillips, W. J. 2004. Vehicle traffic microsimulator for ad hoc networks research. In Proceedings of International Workshop on Wireless AdHoc Networks 2004. IEEE, Oulu, Finland.

[3] Arvelo, E. C. 2003. Open-loop power control based on estimations of packet error rate in a bluetooth radio. In Proceedings of Wireless Communications and Networking 2003, vol.3. 1465-1469.

[4] Ashton, W. D. 1966. The theory of road traffic flow. London, New York, Methuen, Wiley.

[5] Chen, Z. D., Kung, H. T. and Vlah, D. 2001. Ad hoc relay wireless networks over moving vehicles on highways. In Proceedings of the 2nd ACM International Symposium on Mobile Ad Hoc Networking $\backslash \&$ Computing, Long Beach, CA, USA, 247-250.

[6] Cheng, Y. -. and Robertazzi, T. G. 1989. Critical connectivity phenomena in multihop radio models. IEEE Transactions on Communications, 37, 7, 770-777.

[7] Desai, M. and Manjunath, D. 2002. On the connectivity in finite ad hoc networks. IEEE Communications Letters, 6, 10, 437-439.

[8] Dousse, O., Thiran, P. and Hasler, M. 2002. Connectivity in ad-hoc and hybrid networks. In Proceedings of Twenty-First Annual Joint Conference of IEEE Computer and Communications Societies, vol.2, 1079-1088.

[9] Füßler, H., Mauve, M., Hartenstein, H., Vollmer, D. and Käsemann, M. 2002. A comparison of routing strategies in vehicular ad-hoc networks. [Electronic version]. Reihe informatik. Retrieved July 22, 2005 from http://www.cn.uniduesseldorf.de/publications/library/Fuessler2002b.pdf

[10] Gomez, J. and Campbell, A. T. 2004. A case for variablerange transmission power control in wireless multihop networks. In Proceedings of the Twenty-Third Annual Joint Conference of IEEE Computer and Communications Societies, vol.2. 1425-1436 vol.2.

[11] Haberman, R. 1977. Mathematical models : Mechanical vibrations, population dynamics, and traffic flow : An introduction to applied mathematics. Englewood Cliffs, N.J., Prentice-Hall.

[12] Hall, F. L. 1997. Traffic stream characteristics. In Traffic flow theory: A state of the art report - revised monograph on traffic flow theory, N. H. Gartner, C. Messer and A. K. Rathi, Eds., Oak Ridge, Tennessee, Oak Ridge National Laboratory. 
[13] Jost, D. and Nagel, K. 2003. Probabilistic traffic flow breakdown in stochastic car following models. Transportation Research Record, 1852, 152-158.

[14] Kosch, T., Schwingenschlogl, C. and Li Ai. 2002. Information dissemination in multihop inter-vehicle networks. In Proceedings of IEEE 5th International Conference on Intelligent Transportation Systems. 685-690.

[15] Krunz, M., Muqattash, A. and Sung-Ju Lee. 2004. Transmission power control in wireless ad hoc networks: Challenges, solutions and open issues. IEEE Network, 18, 5, 8-14.

[16] Kuhne, R. and Michalopoulos, P. 1997. Continuum flow models. In Traffic flow theory: A state of the art report revised monograph on traffic flow theory, N. H. Gartner, C. Messer and A. K. Rathi, Eds., Oak Ridge, Tennessee, Oak Ridge National Laboratory.

[17] Li, N., Hou, J. C. and Sha, L. 2003. Design and analysis of an MST-based topology control algorithm. In Proceedings of IEEE Twenty-Second Annual Joint Conference of the IEEE Computer and Communications Societies, vol.3. 1702-1712.

[18] Nagatani, T. 2002. The physics of traffic jams. Reports on Progress in Physics, 65, 9, 1331-1386.

[19] Nagel, K., Wagner, P. and Woesler, R. 2003. Still flowing: Approaches to traffic flow and traffic jam modeling. Operations Research, 51, 5, 681-710.

[20] Penrose, M. D. 1997. The longest edge of the random minimal spanning tree. The Annals of Applied Probability, 7, 2, 340-361.
[21] Pipes, L. A. 1953. An operational analysis of traffic dynamics. Journal of Applied Physics, 24, 3, 274-281.

[22] Pivovarov, E. Traffic jams. Retrieved March 22, 2005 from http://physics.ucsd.edu/ epivovar/traffic.htm

[23] Ramanathan, R. and Rosales-Hain, R. 2000. Topology control of multihop wireless networks using transmit power adjustment. In Proceedings of IEEE Nineteenth Annual Joint Conference of the IEEE Computer and Communications Societies, vol.2, 404-413.

[24] Rothery, R. W. 1997. Car-following models. In Traffic flow theory: A state of the art report - revised monograph on traffic flow theory, N. H. Gartner, C. Messer and A. K. Rathi, Eds., Oak Ridge, Tennessee, Oak Ridge National Laboratory.

[25] Rudack, M., Meincke, M. and Lott, M. 2002. On the dynamics of ad hoc networks for inter vehicles communications (IVC). In Proceedings of the 2002 International Conference on Wireless Networks. Las Vegas, USA.

[26] Rudack, M., Meincke, M., Jobmann, K. and Lott, M. 2003. On traffic dynamical aspects of inter vehicle communications (IVC). In Proceedings of IEEE 58th Vehicular Technology Conference, vol.5. 3368-3372.

[27] Santi, P. and Blough, D. M. 2002. An evaluation of connectivity in mobile wireless ad hoc networks. In Proceedings of International Conference on Dependable Systems and Networks. 89-98. 